\title{
Application of the Improved Complex Normal Form Method Based on He's Energy Balance Method to a Noise Included Nonlinear Oscillator
}

\author{
Gen Ge and Zheng Chao Yun \\ School of Mechanical Engineering, Tianjin Polytechnic University, Tianjin 300192, China \\ Correspondence should be addressed to Gen Ge; gegenroot@126.com
}

Received 8 December 2014; Revised 8 April 2015; Accepted 19 April 2015

Academic Editor: P. Balasubramaniam

Copyright (C) 2015 G. Ge and Z. C. Yun. This is an open access article distributed under the Creative Commons Attribution License, which permits unrestricted use, distribution, and reproduction in any medium, provided the original work is properly cited.

\begin{abstract}
A strongly nonlinear oscillator with nonlinear damping subject to narrow band random excitation is discussed. The deterministic and the stochastic responses are studied, respectively, applying the improved complex normal form method based on He's energy balance method. The accuracy of detecting of the response frequency has been improved significantly. The behavior of steady state responses together with the stability is analyzed by the moment method. The numerical simulation results are consistent with theoretical analysis. When the noise intensity is small enough, the analytical result given by the moment method agrees with the simulation quite well.
\end{abstract}

\section{Introduction}

Strongly nonlinear vibration problems have drawn accelerating interest in the past two decades. People have explored various approximate methods to handle the strongly nonlinear problems. A number of them have been developed dramatically and widely applied in analysis of deterministic response problems of nonlinear systems. Actually, some of the approximate methods for deterministic response problems of nonlinear systems can be extended to random response problems. Recently, the modified Lindstedt-Poincare method (MLP) and the multiple time scale method [1-6] are the most popular ways to study noise included nonlinear problems. The basic issue of the two methods is utilizing an undetermined frequency to construct a new expanding parameter which is small enough to change strongly nonlinear systems into weak ones for convenience of using perturbing approaches. Impressive studies have been proposed by Rong and Xu et al. [7-11], and a Duffing-Rayleigh type strongly nonlinear system subject to narrow band noise is studied in detail. In [11], a parameter transformation $\alpha=\varepsilon u_{0}^{2} /\left(4+3 \varepsilon u_{0}^{2}\right)$ combined with multiple time scale method is used to change a strongly nonlinear problem into a weak nonlinear problem, where $\varepsilon$ is a mark denoting the strength of the nonlinear terms which are not small and $u_{0}$ is the amplitude of the first mode of the system. This type of parameter transformation is firstly used by Burton $[12,13]$ to solve deterministic strongly nonlinear problems. In $[9,10]$, another type of parameter transformation $\alpha=\varepsilon \omega_{1} /\left(\omega_{0}^{2}+\varepsilon \omega_{1}\right)$ is introduced, where the undetermined frequency is expressed as $\omega_{10}^{2}=\omega_{0}^{2}+\varepsilon \omega_{1}$ and $\omega_{0}^{2}$ denotes the fundamental frequency of the system.

The complex normal form method proposed by Nayfeh [14] is a powerful tool to study nonlinear dynamical problems. Leung and Zhang [15] studied a Duffing oscillator's free vibration applying this method. Then Wang and Zhang [16-21] developed this method to deal with bifurcation problems and to detect threshold parameters leading to chaotic motions. Besides that, He's energy balance method is a powerful and convenient tool in detecting the strongly nonlinear oscillators' frequency [22]. The classic feature of this method is constructing the system's Hamilton function and keeping the input and output energy in balance. The striking advantage of this method is that the actual frequency does not need to be expanded into series. In other words, the steady state frequency could be solved as a single variable. Thus, the amount of calculation could be reduced significantly. The final expression of the frequency is expressed as a function containing the steady state amplitude and 
the nonlinear parameters. Even if the nonlinearity is driven remarkably large, the error of the approximate frequency is small enough to be neglected. In the previous study, the nonlinear terms together with the external exciting forces and parametrical exciting forces are considered as the reason of changing the frequency of system. As far as we know, studies about the complex normal form method and He's energy method applied on stochastic nonlinear problems are few.

Although these improvements mentioned above are effective in finding frequency of strongly nonlinear systems, these studies are less than perfect. It should be noticed that in these studies the vibration phase has all been ignored. Furthermore, the accuracy of these methods essentially depends on the choice of initial values. If the initial value is chosen just near the actual amplitude the methods will offer precise enough results. But if the initial value is chosen unluckily far from the first-order amplitude, the results could be totally unacceptable. Therefore, there remains a demand for an approach to calculating the frequency, amplitude, and phase of system's responses simultaneously.

The purpose of this paper we present is attempting to extend the complex normal form method to strongly nonlinear stochastic filed. As an example, a Duffing oscillator under narrow band noise excitation was studied in detail. Firstly, a new expression of the response was introduced to improve the performance of Wang's method [16]. Subsequently, He's energy balance method was applied to offer an extra equation which could be combined to the results obtained by the normal form method. Consequently, the accuracy of the frequency could be considerably improved. Thus, both frequency and phase of the response were obtained at the same time. Based on the obtained frequency, the perturbation and the moment method were used to estimate the statistical character of the response. Finally, numerical simulations were carried out to verify the accuracy of the theoretical analysis.

\section{The Complex Normal Form Method}

We take a Duffing oscillator with nonlinear damping subject to external narrow band noise as an example which is as follows:

$$
\begin{aligned}
\ddot{x}+ & \omega_{0}^{2} x+\varepsilon\left[\beta x^{3}+\left(\mu+\gamma x^{2}\right) \dot{x}\right] \\
& =\varepsilon f \cos (\Omega t+\bar{\delta} W(t)),
\end{aligned}
$$

where $\omega_{0}$ denotes the free frequency, $\beta$ denotes the nonlinear Stiffness coefficient, $\mu, \gamma$ are the linear and nonlinear damping coefficients, $f$ denotes the amplitude of the exciting force, $\Omega$ denotes the center frequency of the exciting force, $W(t)$ denotes the standard Wiener process, $\bar{\delta}$ denotes the small strength of the $W(t)$, and the parameter $\varepsilon$ is not small, so we call the system "strongly nonlinear." Such type of equation is widely used in engineering fields such as vibrating beams and plates with nonlinear damping.

In this paper, we focus on the primary resonance. The actual approximate undetermined frequency is set as $\omega_{10}$ and the frequency of the external exciting force $\Omega$ equals $\omega_{10}$. At first, the complex variables $\xi$ and $\eta_{2}$ are introduced to rewrite the system's variables. Thus, the system's displacement $x$ and velocity $\dot{x}$ can be expressed as

$$
\begin{aligned}
& x=\xi+\bar{\xi}, \\
& \dot{x}=\mathrm{i} \omega_{10}(\xi-\bar{\xi}),
\end{aligned}
$$

where $\xi$ is unknown complex variable to be determined and overbar denotes the conjugation.

The external force $f \cos (\Omega t+\bar{\delta} W(t))$ can be rewritten as

$$
f \frac{1}{2}\left(\eta_{2}+\bar{\eta}_{2}\right)
$$

where $\eta_{2}=\mathrm{e}^{\mathrm{i}\left[\omega_{10} t+\bar{\delta} W(t)\right]}$ and $\bar{\eta}_{2}=\mathrm{e}^{-\mathrm{i}\left[\omega_{10} t+\bar{\delta} W(t)\right]}$. Solving (2) for $\xi, \bar{\xi}$ yields

$$
\begin{aligned}
& \xi=\frac{1}{2}\left(x-\frac{\mathrm{i}}{\omega_{10}} \dot{x}\right), \\
& \bar{\xi}=\frac{1}{2}\left(x+\frac{\mathrm{i}}{\omega_{10}} \dot{x}\right) .
\end{aligned}
$$

Taking differentiation to the first function in (4) with respect to time $t$ and considering (1) and (3), one obtains

$$
\begin{aligned}
\xi^{\prime} & =\frac{\mathrm{i}}{2 \omega_{10}}\left[\beta \varepsilon(\xi+\bar{\xi})^{3}+(\xi+\bar{\xi}) \omega_{0}^{2}\right. \\
& +\mathrm{i} \varepsilon(\xi+\bar{\xi})\left(\mu+\gamma(\xi+\bar{\xi})^{2}\right) \omega_{10}+(\xi-\bar{\xi}) \omega_{10}^{2} \\
& \left.-\frac{1}{2} f \varepsilon\left(\eta_{2}+\bar{\eta}_{2}\right)\right] .
\end{aligned}
$$

The basic issue of the complex normal form method is introducing a series of nearly identified transformation. To simplify (5), we introduce a near-identity transformation from $\xi$ to $\eta_{1}$ in the form

$$
\begin{aligned}
& \xi=\eta_{1}+h_{1}\left(\eta_{1}, \bar{\eta}_{1}, \eta_{2}, \bar{\eta}_{2}\right)+h_{2}+h_{3}, \\
& \bar{\xi}=\bar{\eta}_{1}+\bar{h}_{1}\left(\eta_{1}, \bar{\eta}_{1}, \eta_{2}, \bar{\eta}_{2}\right)+\bar{h}_{2}+\bar{h}_{3},
\end{aligned}
$$

where

$$
\begin{aligned}
& h_{n}=\sum_{i=0}^{n} \sum_{j=0}^{n-i} \sum_{k=0}^{n-i-j}\left(a_{i, j, k, n-i-j-k} \eta_{1}^{i} \bar{\eta}_{1}^{j} \eta_{2}{ }^{k} \bar{\eta}_{2}{ }^{n-i-j-k}\right), \\
& \bar{h}_{n}=\sum_{i=0}^{n} \sum_{j=0}^{n-i} \sum_{k=0}^{n-i-j}\left(\bar{a}_{i, j, k, n-i-j-k} \eta_{1}^{j} \bar{\eta}_{1}^{i} \eta_{2}{ }^{n-i-j-k} \bar{\eta}_{2}{ }^{k}\right),
\end{aligned}
$$

where $a_{i, j, k, n-i-j-k}$ are coefficients to be determined. For example,

$$
\begin{aligned}
h_{1}= & a_{1,0,0,0} \eta_{1}+a_{0,1,0,0} \bar{\eta}_{1}+a_{0,0,1,0} \eta_{2}+a_{0,0,0,1} \bar{\eta}_{2}, \\
h_{2}= & a_{2,0,0,0} \eta_{1}^{2}+a_{0,0,2,0} \eta_{2}^{2}+a_{0,2,0,0} \bar{\eta}_{1}^{2}+a_{0,0,0,2} \bar{\eta}_{2}^{2} \\
& +a_{1,1,0,0} \eta_{1} \bar{\eta}_{1}+a_{0,0,1,1} \eta_{2} \bar{\eta}_{2}+a_{1,0,1,0} \eta_{1} \eta_{2} \\
& +a_{0,1,0,1} \bar{\eta}_{1} \bar{\eta}_{2}+a_{0,1,1,0} \eta_{2} \bar{\eta}_{1}+a_{1,0,0,1} \eta_{1} \bar{\eta}_{2} .
\end{aligned}
$$


Substituting (6) and (7) into (5) yields a new equation for $\dot{\eta}_{1}$. Terms proportional to $\eta_{1}, \eta_{2}, \eta_{1}^{2} \bar{\eta}_{1}, \eta_{2}^{2} \bar{\eta}_{2}, \eta_{1}^{2} \bar{\eta}_{2}, \eta_{2}^{2} \bar{\eta}_{1}, \eta_{1} \eta_{2} \bar{\eta}_{1}$, $\eta_{1} \eta_{2} \bar{\eta}_{2}$ are resonance terms and hence cannot be eliminated while all the remaining terms should be eliminated. By eliminating the nonresonance terms of for $\dot{\eta}_{1}$, the simplest possible form gives

$$
\begin{aligned}
\dot{\eta}_{1}= & \frac{\left(\omega_{0}^{2}+\omega_{10}^{2}\right)}{-\varepsilon \mu-2 \mathrm{i} \omega_{10}} \eta_{1}+\frac{\left(3 \beta \varepsilon+\mathrm{i} \gamma \varepsilon \omega_{10}\right)}{-\varepsilon \mu-2 \mathrm{i} \omega_{10}} \eta_{1}^{2} \bar{\eta}_{1} \\
& -\frac{f \varepsilon}{-2 \varepsilon \mu-4 \mathrm{i} \omega_{10}} \eta_{2} .
\end{aligned}
$$

By now, similar work has been presented by Zhang and Wang [16]. The key technique of our work is using a new expression of approximate solution instead of the expression given in [16-21]. Considering the first-order guess of system's response must include both frequency and phase variation, the approximate solution of system (1) with $\bar{\delta}=0$ could be expressed as

$$
x=a(t) \cos \left[\omega_{10} t\right]+b(t) \sin \left[\omega_{10} t\right] .
$$

Thus $\eta_{1}$ and $\bar{\eta}_{1}$ could be expressed as follows:

$$
\begin{aligned}
& \eta_{1}=(1 / 2) a \mathrm{e}^{\mathrm{i} \omega_{10} t}-(1 / 2) \mathrm{i} b \mathrm{e}^{\mathrm{i} \omega_{10} t}, \bar{\eta}_{1}=(1 / 2) a \mathrm{e}^{-\mathrm{i} \omega_{10} t}+ \\
& (1 / 2) \mathrm{i} b \mathrm{e}^{-\mathrm{i} \omega_{10} t}, \text { where } \eta_{1}+\bar{\eta}_{1}=a \cos \left(\omega_{10} t\right)+b \sin \left(\omega_{10} t\right) .
\end{aligned}
$$

Another expression of (10) is

$$
x=A(t) \cos \left[\omega_{10} t+\varphi\right],
$$

where $A(t)=\sqrt{a^{2}+b^{2}}, \tan \varphi=a / b$.

One should notice that the first-order guess of solution expressed as (11) is different from Zhang [12]. In that study, the first-order guess is given as $\eta_{1}=(1 / 2) \mathrm{e}^{\mathrm{i} \omega_{10} t} a, \bar{\eta}_{1}=$ $(1 / 2) a \mathrm{e}^{-\mathrm{i} \omega_{10} t}$. The new expression ensures the phase existing.

Thus, our job is calculating the relationship of the three undetermined variables $\omega_{10}, a$, and $b$.

By substituting (10) and (3) into (9), one obtains

$$
\begin{aligned}
& \frac{1}{2} \frac{d a}{d t}-\frac{\mathrm{i}}{2} \frac{d b}{d t}+\frac{1}{2} \mathrm{i} a \omega_{10} \\
& +\frac{1}{2} b \omega_{10}=\frac{-4 f+3(a-\mathrm{i} b)^{2}(a+\mathrm{i} b) \beta \varepsilon+4(a-\mathrm{i} b) \omega_{0}^{2}+\mathrm{i}(a-\mathrm{i} b)^{2}(a+\mathrm{i} b) \gamma \varepsilon \omega_{10}+4(a-\mathrm{i} b) \omega_{10}^{2}}{8\left(\varepsilon \mu+2 \mathrm{i} \omega_{10}\right)} .
\end{aligned}
$$

Multiplying both sides of (12) with $8\left(\varepsilon \mu+2 \mathrm{i} \omega_{10}\right)$ and separating the real and image parts yield

$$
\begin{aligned}
& \frac{d a}{d t} \\
& =\frac{\left\{\left[4 f-3 a\left(a^{2}+b^{2}\right) \beta\right] \varepsilon^{2} \mu-b \varepsilon\left[4 \varepsilon \mu^{2}+a^{2}(-6 \beta+\gamma \varepsilon \mu)+b^{2}(-6 \beta+\gamma \varepsilon \mu)\right] \omega_{10}-2 a \varepsilon\left(a^{2} \gamma+b^{2} \gamma+2 \mu\right) \omega_{10}^{2}-8 b \omega_{10}^{3}+\omega_{0}^{2}\left(-4 a \varepsilon \mu+8 b \omega_{10}\right)\right\}}{\left[4\left(\varepsilon^{2} \mu^{2}+4 \omega_{10}^{2}\right)\right]} \\
& \frac{d b}{d t} \\
& =\frac{\left\{-3 b\left(a^{2}+b^{2}\right) \beta \varepsilon^{2} \mu+\varepsilon\left[8 f+a\left(4 \varepsilon \mu^{2}+a^{2}(-6 \beta+\gamma \varepsilon \mu)+b^{2}(-6 \beta+\gamma \varepsilon \mu)\right)\right] \omega_{10}-2 b \varepsilon\left(a^{2} \gamma+b^{2} \gamma+2 \mu\right) \omega_{10}^{2}+8 a \omega_{10}^{3}-\omega_{0}^{2}\left(4 b \varepsilon \mu+8 a \omega_{10}\right)\right\}}{\left[4\left(\varepsilon^{2} \mu^{2}+4 \omega_{10}^{2}\right)\right]} .
\end{aligned}
$$

Until then, the full expressions of $d a / d t$ and $d b / d t$ are proposed in (13). Theoretically, the steady state solution can be obtained by letting $d a / d t=d b / d t=0$.

By now the method has not been finished. As we know, there are three unknown variables $\omega_{10}, a$, and $b$ to be determined. But we only have two equations. Chen $[1,2]$ offered an approach to solve this problem. With the initial displacement value $x=a$ given previously, the variables $\omega_{10}, b$ can be calculated by (13) subsequently. However, using this approach has limitation to some extent. As mentioned in Introduction, if the initial displacement value $a$ is chosen correctly, the solution will be precise enough. But if the initial displacement is not given properly, (13) may offer nonreal roots or totally unacceptable roots. Thus, one extra equation is needed to solve unknown variables $\omega_{10}, a$, and $b$ simultaneously.

\section{He's Energy Balance Method}

The so-called energy balance method proposed by He [22] has been used and developed by many researchers to determine frequency of nonlinear oscillations in view of the fact that it is very effective and convenient. The striking issue of He's method is building a Hamilton and then making the input and output of it kept in balance. The validity has 
been verified in previous studies [23-27]. Thus, we take this approach to build an extra equation so that (13) can be solved.

The Hamilton of system (1) is given as follows:

$$
\begin{aligned}
H= & \frac{1}{2} \dot{x}^{2}+\frac{1}{2} \omega_{0}^{2} x^{2}+\frac{1}{4} \beta x^{4}+\left(\mu x+\frac{1}{3} \gamma x^{3}\right) \dot{x} \\
& -f \cos \left(\omega_{10} t\right) x .
\end{aligned}
$$

Substituting the assumed first-order approximate solution (10) into (14) and letting $\omega_{10} t \rightarrow \pi / 4$ and $\omega_{10} t \rightarrow 0$, respectively, yield

$$
\begin{aligned}
& H_{\omega_{10} t \rightarrow \pi / 4} \\
& \quad=\frac{1}{16}\left((a+b)\left(a^{3} \beta+3 a^{2} b \beta+3 a b^{2} \beta+b^{3} \beta-8 f \varepsilon\right)\right. \\
& \quad+4(a+b)^{2} \omega_{0}^{2} \\
& \quad-4\left(a^{2}-b^{2}\right) \varepsilon\left(a^{2} \gamma+2 a b \gamma+b^{2} \gamma+2 \mu\right) \omega_{10} \\
& \left.\quad+4(a-b)^{2} \omega_{10}^{2}\right) \\
& H_{\omega_{10} t \rightarrow 0}=\frac{a^{4} \beta}{4}-a f \mathcal{E}+\frac{1}{2} a^{2} \omega_{0}^{2}+b \varepsilon\left(a^{3} \gamma+a \mu\right) \omega_{10} \\
& \quad+\frac{1}{2} b^{2} \omega_{10}^{2} .
\end{aligned}
$$

The residual can be expressed as $R=H_{\omega_{10} t \rightarrow \pi / 4}-H_{\omega_{10} t \rightarrow 0}$. Then, based on Galerkin-Petrov method [28], one equation is obtained as

$$
\begin{aligned}
& \int_{0}^{T / 4} R \cos \left(\omega_{10} t\right) d t=\frac{1}{16 \omega_{10}}\left\{-3 a^{4} \beta+4 a^{3} b \beta\right. \\
& +6 a^{2} b^{2} \beta+4 a b^{3} \beta+b^{4} \beta+8 a f \mathcal{\varepsilon}-8 b f \varepsilon+4\left(-a^{2}\right. \\
& \left.+2 a b+b^{2}\right) \omega_{0}^{2}+4 \omega_{10}\left[\left(a^{4}+6 a^{3} b-2 a b^{3}-b^{4}\right) \gamma \varepsilon\right. \\
& \left.\left.\quad-2\left(a^{2}+2 a b-b^{2}\right) \varepsilon \mu+\left(a^{2}-2 a b-b^{2}\right) \omega_{10}\right]\right\},
\end{aligned}
$$

where $T=2 \pi / \omega_{10}$ means the period.

Combing (17) with (13), three equations can be solved numerically to determine the three approximate variables $\omega_{1}$, $a$, and $b$.

\section{Perturbation Procedures}

By introducing a detuning parameter $\sigma$ into $\Omega^{2}$, we attempt to investigate how the response of the noise included strongly nonlinear system (1) changes by the detuning parameter $\sigma$. Considering the parameter transformation $\alpha=\varepsilon \omega_{1} /\left(\omega_{0}^{2}+\right.$ $\left.\varepsilon \omega_{1}\right)$ introduced in $[9,10]$ and the undetermined frequency expressed as $\omega_{10}^{2}=\omega_{0}^{2}+\varepsilon \omega_{1}$, the exciting frequency is expressed as

$$
\Omega^{2}=\omega_{10}^{2}(1+\sigma \alpha)=\frac{\omega_{0}^{2}(1+\sigma \alpha)}{1-\alpha} .
$$

A new form of $\eta_{1}$ is given as

$$
\eta_{1}=\frac{1}{2} a(t) \mathrm{e}^{\mathrm{i}\left[\omega_{10} t+\varphi(t)\right]}
$$

which is different from (10).

Substituting (3) and (19) into (9) and separating the real and image parts yield

$$
\begin{aligned}
& \frac{d a}{d t}=-\frac{\varepsilon\left[2 \omega_{10}^{2}\left(a^{3} \gamma+2 a \mu\right)+\mu \varepsilon\left(3 a^{3} \beta-4 f \cos (\delta W(t)-\varphi)\right)+4 a \mu \omega_{0}^{2}-8 f \omega_{10} \sin (\delta W(t)-\varphi)\right]}{4\left(4 \omega_{10}^{2}+\mu^{2} \epsilon^{2}\right)} \\
& \frac{d \varphi}{d t}=\frac{\omega_{10}\left[8 a \omega_{0}^{2}-\varepsilon\left(a^{3}(\gamma \mu \epsilon-6 \beta)+4 a \mu^{2} \varepsilon+8 f \cos (\delta W(t)-\varphi)\right)\right]-8 a \omega_{10}^{3}+4 f \mu \varepsilon^{2} \sin (\delta W(t)-\varphi)}{4 a\left(4 \omega_{10}^{2}+\mu^{2} \epsilon^{2}\right)} .
\end{aligned}
$$

Substituting (18) into (20), expanding (20) in Taylor series with respect to $\alpha$, and then ignoring the high order terms, one obtains

$$
\begin{aligned}
& \frac{d a}{d t}=\frac{\left(4 f \sin [\bar{\delta} W(t)-\varphi]-a^{3} \gamma \omega_{10}-4 a \mu \omega_{10}\right) \omega_{10} \alpha}{8 \omega_{1}} \\
& \frac{d \varphi}{d t} \\
& =\frac{\left(3 a^{3} \beta-4 f \cos [\bar{\delta} W(t)-\varphi]-4 a \omega_{1}-4 a \sigma \omega_{1}\right) \omega_{10} \alpha}{8 a \omega_{1}} .
\end{aligned}
$$

Introducing a time scale transformation $T_{1}=\omega_{10} \alpha t$, (21) can be rewritten as

$$
\begin{aligned}
\frac{d a}{d T_{1}}= & -\frac{f}{2 \omega_{1}} \sin \left[\delta W\left(T_{1}\right)-\varphi\right]-\frac{a^{3} \gamma \omega_{10}}{8 \omega_{1}}-\frac{a \mu \omega_{10}}{2 \omega_{1}}, \\
\frac{d \varphi}{d T_{1}}= & -\frac{1}{2}(1+\sigma)+\frac{3 a^{2} \beta}{8 \omega_{1}} \\
& -\frac{f}{2 a \omega_{1}} \cos \left[\delta W\left(T_{1}\right)-\varphi\right]
\end{aligned}
$$

where $\delta=\bar{\delta} / \sqrt{\omega_{10} \alpha}$, as we know for Wiener process $W(t)$ which is a mathematical tool to describe famous Brown's 
motion; there exists $E[W(t)]=0, E\left[W^{2}(t)\right]=t$, where $E[\cdot]$ denotes the mathematical expectation.

Rewrite (22) as the following Ito type equation, with the parameters $\gamma^{\prime}=\gamma \omega_{10} / \omega_{0}, \mu^{\prime}=\mu \omega_{10} / \omega_{0}$, and $\eta=\varphi-\delta W^{\prime}\left(T_{1}\right)$ :

$$
\begin{aligned}
d a= & \left(-\frac{1}{2} \mu^{\prime} a-\frac{1}{8} \gamma^{\prime} a^{3}-\frac{1}{2 \omega_{1}} f \sin \eta\right) d T_{1} \\
d \eta= & \left(-\frac{\sigma+1}{2}+\frac{3}{8 \omega_{1}} \beta a^{2}-\frac{1}{2 a \omega_{1}} f \cos \eta\right) d T_{1} \\
& -\delta d W\left(T_{1}\right) .
\end{aligned}
$$

The first-order steady state approximate response of noise-free system (1) is expressed as

$x=\bar{a} \cos \left[\omega_{10} t+\bar{\eta}\right](26)$, where $\bar{a}, \bar{\eta}$ are determined by (23). When $d a / d T_{1}=d \eta / d T_{1}=0$ and $\delta=0$, such that

$$
\begin{gathered}
-\frac{1}{2} \mu^{\prime} \bar{a}-\frac{1}{8} \gamma^{\prime} \bar{a}^{3}=\frac{1}{2 \omega_{1}} f \sin \bar{\eta} \\
-\frac{\sigma+1}{2}+\frac{3}{8 \omega_{1}} \beta \bar{a}^{2}=\frac{1}{2 a \omega_{1}} f \cos \bar{\eta}
\end{gathered}
$$

squaring and adding both sides of equations in (24), one obtains the frequency-amplitude response function

$$
\begin{aligned}
& \left(-\frac{1}{2} \mu^{\prime} \bar{a}-\frac{1}{8} \gamma^{\prime} \bar{a}^{3}\right)^{2}+\left(-\frac{\sigma+1}{2} \bar{a}+\frac{3}{8 \omega_{1}} \beta \bar{a}^{3}\right)^{2} \\
& =\frac{1}{4 \omega_{1}^{2}} f^{2} .
\end{aligned}
$$

\section{The Moment Method}

Next, we want to explore the probability density of the responses. Then we discuss the effect of the noise. The response of the system is determined by the steady state probability density function. As the Fokker-Planck-Kolmogorov equation for the response probability density function (PDF) is not analytically solvable, the stochastic moment method [29] is considered alternatively. The moment method is an acceptable backup to study the character of the responses approximately. We set

$$
\begin{aligned}
& a=\bar{a}+\Delta a, \\
& \eta=\bar{\eta}+\Delta \eta,
\end{aligned}
$$

where $\Delta a, \Delta \eta$ are the small perturbation terms; substituting the above equations into (24) and ignoring the high order terms, the linearization equation is expressed as

$$
\begin{aligned}
\frac{d \Delta a}{d T_{1}}= & \left(-\frac{\mu^{\prime}}{2}-\frac{3}{8} \gamma^{\prime} \bar{a}^{2}\right) \Delta a \\
& +\left(\frac{1+\sigma}{2} \bar{a}-\frac{3}{8 \omega_{1}} \beta \bar{a}^{3}\right) \Delta \eta
\end{aligned}
$$

$$
\begin{aligned}
\frac{d \Delta \eta}{d T_{1}}= & \left(-\frac{1+\sigma}{2 \bar{a}}+\frac{9}{8 \omega_{1}} \beta \bar{a}\right) \Delta a \\
& +\left(-\frac{\mu^{\prime}}{2}+\frac{1}{8} \gamma^{\prime} \bar{a}^{2}\right) \Delta \eta-\delta d W\left(T_{1}\right),
\end{aligned}
$$

where $d W(t)$ denotes the standard Gauss white noise.

Taking expectation on both sides of (27) and $d E[\Delta a] /$ $d T_{1}=d E[\Delta \eta] / d T_{1}=0$ one obtains

$$
E[\Delta a]=E[\Delta \eta]=0 .
$$

Thus, it is obvious that $E[a]=\bar{a}, E[\eta]=\bar{\eta}$.

Based on the Floquet theory, the Jacoby matrix is used to judge the stability of the first-order moment, which is expressed as follows:

$$
\operatorname{det}\left[\begin{array}{cc}
\left(-\frac{\mu^{\prime}}{2}-\frac{3}{8} \gamma^{\prime} \bar{a}^{2}\right)-\lambda & \left(\frac{1+\sigma}{2} \bar{a}-\frac{3}{8 \omega_{1}} \beta \bar{a}^{2}\right) \\
\left(-\frac{1+\sigma}{2 \bar{a}}+\frac{9}{8 \omega_{1}} \beta \bar{a}\right) & \left(-\frac{\mu^{\prime}}{2}+\frac{1}{8} \gamma^{\prime} \bar{a}^{2}\right)-\lambda
\end{array}\right]
$$

$=0$.

The characteristic equation can be expressed as $\lambda^{2}+P \lambda+Q=$ 0 , where

$$
\begin{aligned}
P= & \frac{\bar{a}^{2} \gamma^{\prime}}{2}+\mu^{\prime} \\
Q= & \bar{a}^{4}\left(\frac{27 \beta^{2}}{64 \omega_{1}^{2}}-\frac{3 \gamma^{\prime 2}}{64}\right)+\bar{a}^{2}\left[\frac{\gamma^{\prime} \mu^{\prime} \omega_{1}-3 \beta(1+\sigma)}{4 \omega_{1}}\right] \\
& +\frac{1}{4}\left[(1+\sigma)^{2}+\mu^{\prime 2}\right] .
\end{aligned}
$$

The roots of (30) are

$$
\lambda_{1,2}=\frac{-P \pm \sqrt{P^{2}-4 Q}}{2} .
$$

Obviously, the stable condition of (27) without noise is $P>0$, $Q>0$, which means the solution is realizable by numerical simulation.

Then the moment method is applied to study the secondorder moment of the system's responses. Similarly, we have the obvious results $d E\left[\Delta a^{2}\right] / d T_{1}=d E\left[\Delta \eta^{2}\right] / d T_{1}=$ $d E[\Delta a \Delta \eta] / d T_{1}=0$. Applying Ito's rule [30] of stochastic differentiation, one obtains

$$
\begin{aligned}
& \left(-\frac{3}{4} \bar{a}^{2} \gamma^{\prime}-\mu^{\prime}\right) E\left[\Delta a^{2}\right] \\
& \quad+\left[\bar{a}(1+\sigma)-\frac{3 \bar{a}^{3} \beta}{4 \omega_{1}}\right] E[\Delta a \Delta \eta]=0 .
\end{aligned}
$$




$$
\begin{aligned}
& \left(-\frac{1+\sigma}{\bar{a}}+\frac{9 \bar{a} \beta}{4 \omega_{1}}\right) E[\Delta a \Delta \eta] \\
& +\left(\frac{\bar{a}^{2} \gamma^{\prime}}{4}-\mu^{\prime}\right) E\left[\Delta \eta^{2}\right]=-\delta^{2} \\
& \left(-\frac{1+\sigma}{2 \bar{a}}+\frac{9 \bar{a} \beta}{8 \omega_{1}}\right) E\left[\Delta a^{2}\right]
\end{aligned}
$$

$$
\begin{aligned}
& +\left(-\frac{1}{4} \bar{a}^{2} \gamma^{\prime}-\mu^{\prime}\right) E[\Delta a \Delta \eta] \\
& +\left(\frac{\bar{a}(1+\sigma)}{2}-\frac{3 \bar{a}^{3} \beta}{8 \omega_{1}}\right) E\left[\Delta \eta^{2}\right]=0 .
\end{aligned}
$$

The solutions of (32) are

$$
\begin{aligned}
E\left[\Delta a^{2}\right] & =\frac{\bar{a}^{2} \delta^{2}\left[3 \bar{a}^{2} \beta-4(1+\sigma) \omega_{1}\right]^{2}}{32 \omega_{1}^{2}\left(\bar{a}^{2} \gamma^{\prime}+4 \mu^{\prime}\right)\left\{\bar{a}^{4}\left(27 \beta^{2} / 64 \omega_{1}^{2}-3 \gamma^{\prime 2} / 64\right)+\bar{a}^{2}\left[\gamma^{\prime} \mu^{\prime} / 4-3 \beta(\sigma+1) / 4 \omega_{1}\right]+(1 / 4)\left[\left(\mu^{\prime}\right)^{2}+(\sigma+1)^{2}\right]\right\}} \\
E[\Delta a \Delta \eta] & =\frac{\delta^{2} \bar{a} \omega_{1}\left[3 \bar{a}^{2} \beta-4(1+\sigma) \omega_{1}\right]\left(3 \bar{a}^{2} \gamma^{\prime}+4 \mu^{\prime}\right)}{32 \omega_{1}^{2}\left(\bar{a}^{2} \gamma^{\prime}+4 \mu^{\prime}\right)\left\{\bar{a}^{4}\left(27 \beta^{2} / 64 \omega_{1}^{2}-3 \gamma^{\prime 2} / 64\right)+\bar{a}^{2}\left[\gamma^{\prime} \mu^{\prime} / 4-3 \beta(\sigma+1) / 4 \omega_{1}\right]+(1 / 4)\left[\left(\mu^{\prime}\right)^{2}+(\sigma+1)^{2}\right]\right\}} \\
E\left[\Delta \eta^{2}\right] & =\frac{\delta^{2}\left\{a^{4}\left(27 \beta^{2}+6 \omega_{1}^{2} \gamma^{\prime 2}\right)+a^{2}\left[32 \omega_{1}^{2} \gamma^{\prime} \mu^{\prime}-48 \beta(\sigma+1) \omega_{1}\right]+16 \omega_{1}^{2}\left[2 \mu^{\prime 2}+(\sigma+1)^{2}\right]\right\}}{32 \omega_{1}^{2}\left(\bar{a}^{2} \gamma^{\prime}+4 \mu^{\prime}\right)\left\{\bar{a}^{4}\left(27 \beta^{2} / 64 \omega_{1}^{2}-3 \gamma^{\prime 2} / 64\right)+\bar{a}^{2}\left[\gamma^{\prime} \mu^{\prime} / 4-3 \beta(\sigma+1) / 4 \omega_{1}\right]+(1 / 4)\left[\left(\mu^{\prime}\right)^{2}+(\sigma+1)^{2}\right]\right\}} .
\end{aligned}
$$

One obtains

$$
\begin{aligned}
& E\left[a^{2}\right]=E\left[(\bar{a}+\Delta a)^{2}\right]=\bar{a}^{2}+E\left[\Delta a^{2}\right] \\
& E\left[\eta^{2}\right]=E\left[(\bar{\eta}+\Delta \eta)^{2}\right]=\bar{\eta}^{2}+E\left[\Delta \eta^{2}\right] .
\end{aligned}
$$

Form (33), it is easy to see that, for $E\left[\Delta a^{2}\right] \geq 0, E\left[\Delta \eta^{2}\right] \geq 0$, there must be

$$
\begin{gathered}
\bar{a}^{4}\left(\frac{27 \beta^{2}}{64 \omega_{1}^{2}}-\frac{3 \gamma^{\prime 2}}{64}\right)+\bar{a}^{2}\left[\frac{\gamma^{\prime} \mu^{\prime} \omega_{1}-3 \beta(1+\sigma)}{4 \omega_{1}}\right] \\
+\frac{1}{4}\left[(1+\sigma)^{2}+\mu^{\prime 2}\right]>0 .
\end{gathered}
$$

Function (35) also means the solutions are stable if the inequality is satisfied (see (30)).

When the noise density is small enough, the response's probability density function can be approximately expressed as the Gauss distribution:

$$
N\left(\bar{a}, E\left[\Delta a^{2}\right]\right)=\frac{1}{\sqrt{2 \pi \sqrt{E\left[\Delta a^{2}\right]}}} \exp \left[-\frac{(a-\bar{a})^{2}}{2 E\left[\Delta a^{2}\right]}\right] .
$$

\section{Results and Numerical Simulation}

Let the coefficients be $\omega_{0}=2, \varepsilon=1, f=1, \mu=2$, $\gamma=1$, and $\beta=8$. Firstly, we study the noise-free system. Combing (13) and (17), the undetermined variables were obtained as $a=-0.0218, b=0.2263, \omega_{10}=2.1752$, and $\omega_{1}=0.7313$. Thus the amplitude was calculated as $A=$ $\sqrt{a^{2}+b^{2}}=0.2273$. To compare the accuracy of this method and the improved LP method, the results were displayed as follows, respectively. With the initial displacement $a$ chosen as $=-0.02$, the variables $b$ and $\omega_{10}$ were given by the improved LP method as $b=0.2465, \omega_{10}=2.1665$, and $\omega_{1}=0.6939$.
As seen in Figure 1, it is obvious that the accuracy of the improved complex normal form method is much better than the improved LP method. In Figure 1(a) one can see that the actual result and the approximate result are so close to each other that the difference can hardly be found. On the other hand, the result given by the improved LP method is slightly different from the actual result. Furthermore, as mentioned in Introduction, the accuracy of the improved LP method depends on the initial values. If the initial value $a$ is chosen randomly, the improved LP method may offer nonreal results.

Subsequently, we study the noise included system. By the method proposed by Askari et al. [28], the numerical results of (1) are given. The simulation time is set as $t=400$, and the time step size is set as $\Delta t=0.01$, and four groups of narrow band noises with different intensity $\bar{\delta}$ are imposed to system (1) separately.

Figure 2 shows the phase plots of the last 100 seconds. In Figure 2 the $x$-axis denotes the displacement and the $y$-axis denotes the velocity. With the detuning parameter $\sigma$ chosen as 0 , it is clear that the increasing noise intensity results in the changing of the amplitude. In Figure 2, it is found that the limit cycle presenting the periodical motion can be diffused by the noise. The stronger the strength of noise is, the wider the width of the limit cycle will be. The noise will change the periodical vibration into a quasiperiodic motion.

Obviously, the amplitude of the vibration is varying slightly within some certain range. To explore how the noise influences the amplitude, the amplitudes of the last 150 vibration periods are taken into account. With the help of (27), the average steady state amplitude is solved as $\bar{a}=$ 0.2273 . Then, when $\bar{\delta}$ equals 0.1 and 0.2 separately, $E\left[\Delta a^{2}\right]$ is given by (33) as 0.0078 and 0.015 . With these parameters mentioned above, both the numerical results and the approximate probability density function (36) are illustrated in Figure 3. In Figure 3(a), the numerical simulation agrees with the analytical result very well because the noise intensity 


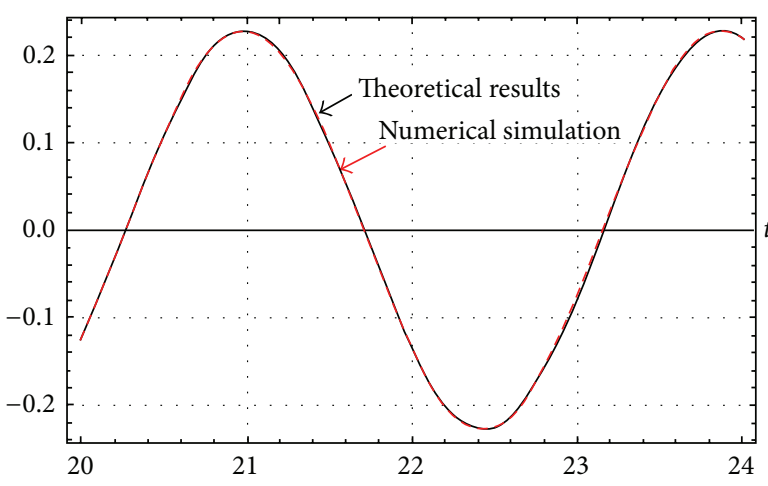

(a)

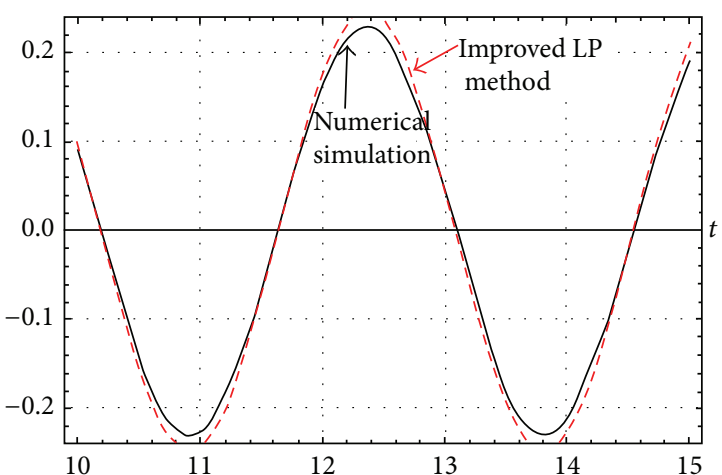

(b)

FIGURE 1: Comparison of the improved complex normal form method and the improved LP method. (a) The improved complex normal form method. (b) The improved LP method.

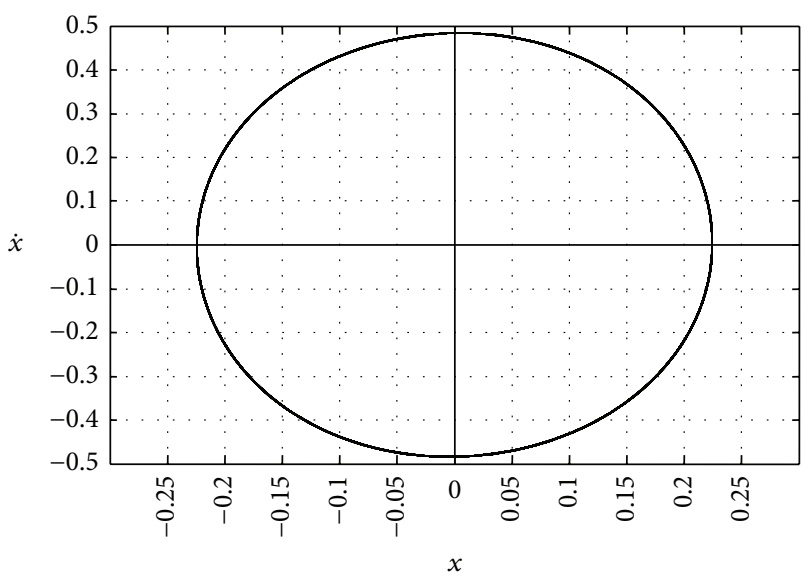

(a)

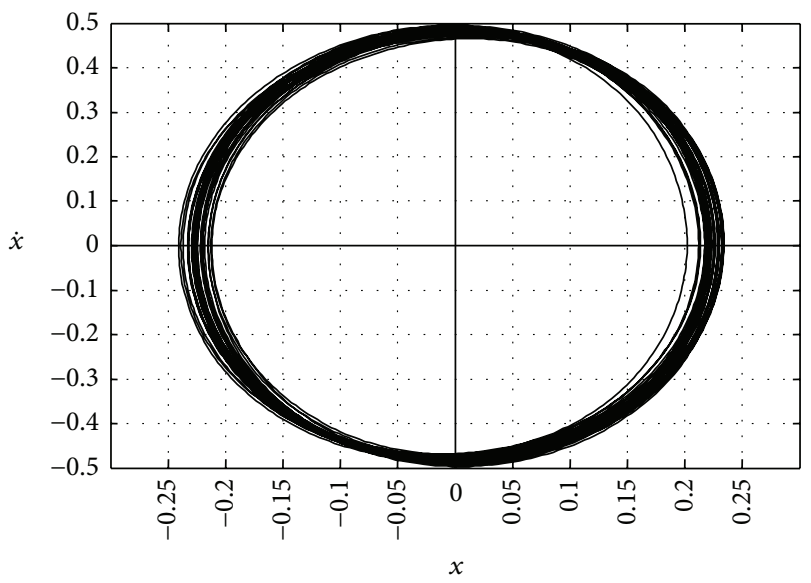

(c)

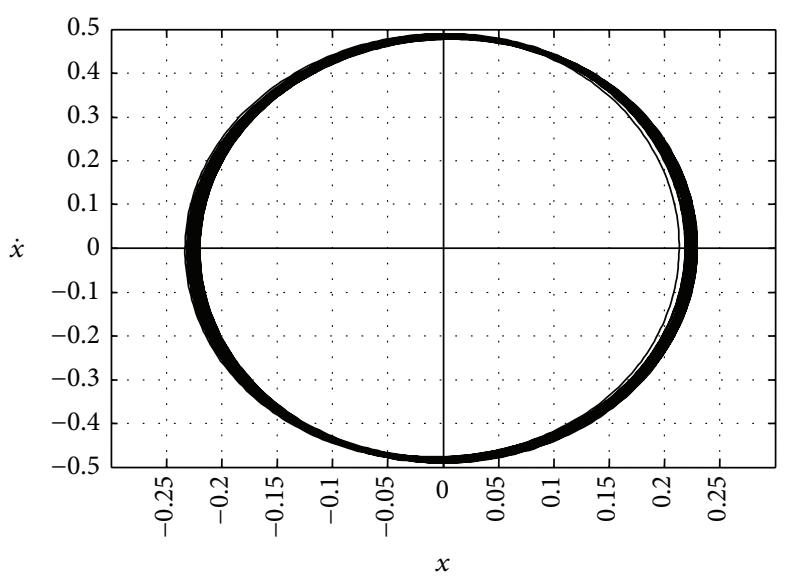

(b)

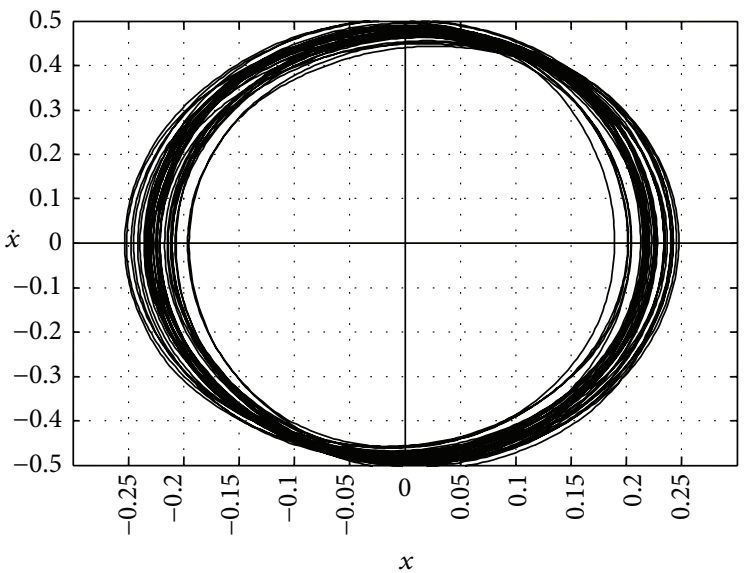

(d)

FIGURE 2: The phase of the vibration: (a) $\bar{\delta}=0$, (b) $\bar{\delta}=0.05$, (c) $\bar{\delta}=0.1$, and (d) $\bar{\delta}=0.2$.

is small enough. Comparing Figures 3(a) and 3(b), we find that the variance solved by (33) analytically is slightly larger than the numerical results with increasing noise density. The larger the noise intensity becomes, the greater the difference of the numerical results between the analytical solutions will be. We could explain the phenomena like this: the moment method is obtained based on linearization equation (27), which means only small variations around the steady state amplitude $\bar{a}$ can be approximately described by the second moment $E\left[\Delta a^{2}\right]$. However, for narrow band noise excitations, 


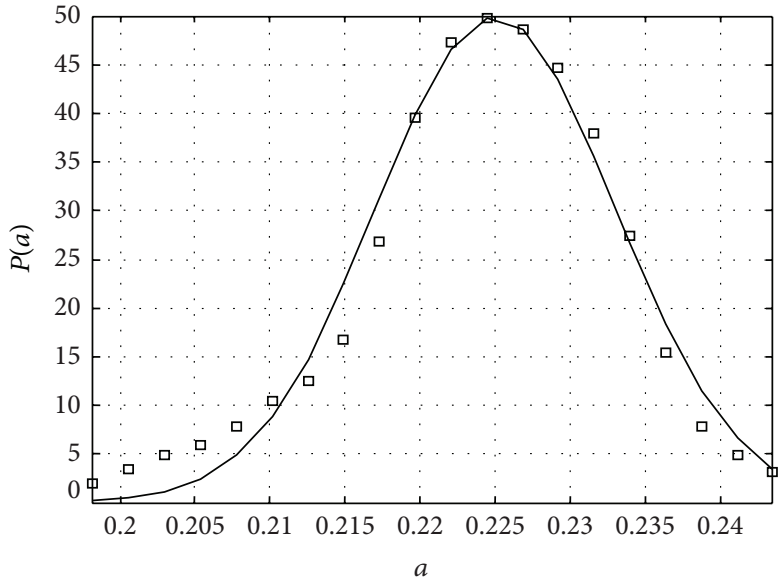

(a)

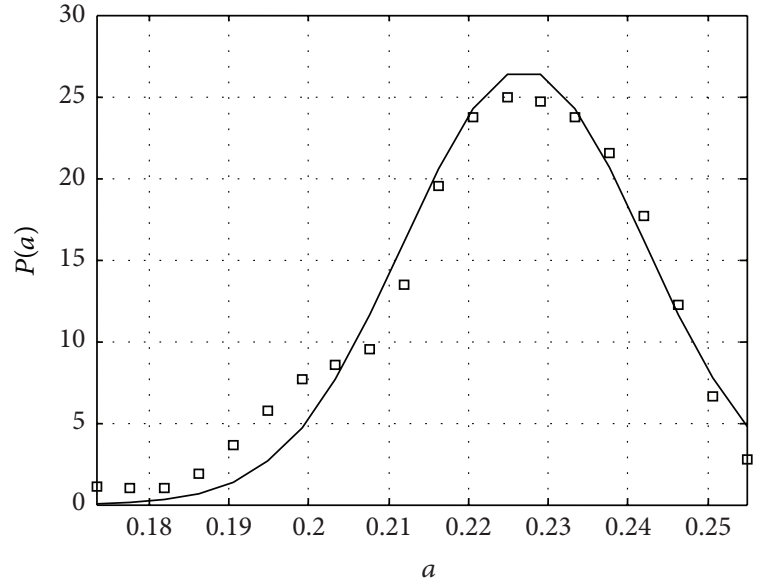

(b)

Figure 3: Probability density of the amplitudes: (a) $\bar{\delta}=0.1$, (b) $\bar{\delta}=0.2$.

the precision of the results obtained by the moment method is acceptable.

\section{Discussions}

A strongly nonlinear system subject to narrow band noise is discussed based on the complex normal form method and the moment method in this paper. Some results are obtained.

(1) For this type of strongly nonlinear equation excited by narrow band noise, the complex normal form which is effective to solve deterministic problems still has good performance in dealing with narrow band noise included systems. After the simplest normal form was determined (see (9)) two different kinds of expression of $\eta_{1}$ were used (see (10) and (19)); the former one leads to an approximate solution which is more precise than the results offered by the MLP method, while the latter one was used to carry out the perturbation procedure.

(2) The main effect of the noise is to change the periodical motion to a quasiperiodic motion. The limit cycle will be diffused by the noise, and the width of the cycle becomes wider when the noise intensity increases. Anyway, the averaged amplitude does not change so much, when the noise intensity is fairly small.

(3) The Fokker-Planck-Kolmogorov (FPK) equation for the response probability density function is our first choice to gain the steady state response of the system. But in this problem, the FPK function is not analytically solvable, so the moment method is used to get the first-order and second-order moments of the steady state responses. As an alternative plan, the moment method performs well when the noise intensity is small enough. It is obvious that the response is of the normal distribution form.

\section{Conflict of Interests}

The authors declare that there is no conflict of interests regarding the publication of this paper.

\section{Acknowledgments}

The authors gratefully acknowledge the support of the Natural Science Foundation of China (NSFC) through Grant nos. 11402186 and 11302144 and Tianjin Research Program of Application Foundation and Advanced Technology through Grant nos. 14JCQNJC05600, 13JCYBJC17900, and 14JCQNJC05300.

\section{References}

[1] S. H. Chen and Y. K. Cheung, "A modified Lindstedt-Poincaré method for a strongly nonlinear system with quadratic and cubic nonlinearities," Shock and Vibration, vol. 3, no. 4, pp. 279285, 1996.

[2] S. H. Chen and Y. K. Cheung, "A modified Lindstedt-Poincaré method for a strongly non-linear two degree-of-freedom system," Journal of Sound and Vibration, vol. 193, no. 4, pp. 751-762, 1996.

[3] K. Richard and G. V. Anand, "Non-linear resonance in strings under narrow band random excitation, part I: planar response and stability," Journal of Sound and Vibration, vol. 86, no. 1, pp. 85-98, 1983.

[4] T. Kapitaniak, "Stochastic response with bifurcations to nonlinear Duffing's oscillator," Journal of Sound and Vibration, vol. 102, no. 3, pp. 440-441, 1985.

[5] S. Rajan and H. G. Davies, "Multiple time scaling of the response of a Duffing oscillator to narrow-band random excitation," Journal of Sound and Vibration, vol. 123, no. 3, pp. 497-506, 1988.

[6] A. H. Nayfeh and S. J. Serhan, "Response statistics of nonlinear systems to combined deterministic and random excitations," International Journal of Non-Linear Mechanics, vol. 25, no. 5, pp. 493-507, 1990.

[7] X. Yang, W. Xu, Z. Sun, and Y. Xu, "Responses of strongly nonlinear oscillator parametrically excited by random narrow-band 
noise," Applied Mathematics and Computation, vol. 171, no. 2, pp. 885-899, 2005.

[8] H.-W. Rong, X.-D. Wang, G. Meng, W. Xu, and T. Fang, "Response of nonlinear oscillator under narrow-band random excitation," Applied Mathematics and Mechanics, vol. 24, no. 7, pp. 817-825, 2003.

[9] J. R. Li, W. Xu, Z. Z. Ren, and Y. Lei, "Maximal Lyapunov exponent and almost-sure stability for stochastic MathieuDuffing systems," Journal of Sound and Vibration, vol. 286, no. 1-2, pp. 395-402, 2005.

[10] H. Rong, W. Xu, and T. Fang, "Principal response of duffing oscillator to combined deterministic and narrow-band random parametric excitation," Journal of Sound and Vibration, vol. 210, no. 4, pp. 483-515, 1998.

[11] H. W. Rong, G. Meng, X. D. Wang, W. Xu, and T. Fang, "Response of a strongly non-linear oscillator to narrowband random excitations," Journal of Sound and Vibration, vol. 266, no. 4, pp. 875-887, 2003.

[12] T. D. Burton, "Nonlinear oscillator limit cycle analysis using a time transformation approach," International Journal of NonLinear Mechanics, vol. 17, no. 1, pp. 7-19, 1982.

[13] T. D. Burton, "A perturbation method for certain non-linear oscillators," International Journal of Non-Linear Mechanics, vol. 19, no. 5, pp. 397-407, 1984.

[14] A. H. Nayfeh, The Method of Normal Forms, Wiley Series in Nonlinear Science, John Wiley \& Sons, New York, NY, USA, 1993.

[15] A. Y. Leung and Q. C. Zhang, "Complex normal form for strongly non-linear vibration systems exemplified by Duffingvan der Pol equation," Journal of Sound and Vibration, vol. 213, no. 5, pp. 907-914, 1998.

[16] W. Wang, Q. C. Zhang, and X. J. Wang, "Application of the undetermined fundamental frequency method for analyzing the critical value of chaos," Acta Physica Sinica, vol. 58, no. 8, pp. 5162-5168, 2009.

[17] W. Wang and Q.-C. Zhang, "Computation of the simplest normal form of a resonant double Hopf bifurcation system with the complex normal form method," Nonlinear Dynamics, vol. 57, no. 1-2, pp. 219-229, 2009.

[18] Q.-C. Zhang, W. Wang, and F.-H. Liu, "High-codimensional static bifurcations of strongly nonlinear oscillator," Chinese Physics B, vol. 17, no. 11, pp. 4123-4128, 2008.

[19] Q. C. Zhang, W. Wang, and X. J. He, "Homoclinic bifurcation of a strongly nonlinear oscillation system by a normal form method," Acta Physica Sinica, vol. 57, no. 9, pp. 5384-5389, 2008.

[20] Q.-C. Zhang, W. Wang, and W.-Y. Li, "Heteroclinic bifurcation of strongly nonlinear oscillator," Chinese Physics Letters, vol. 25, no. 5, pp. 1905-1907, 2008.

[21] L. Fu-Hao, Z. Qi-Chang, and W. Wei, "Analysis of hysteretic strongly nonlinearity for quad iced bundle conductors," Chinese Physics Letters, vol. 27, no. 3, Article ID 034703, 2010.

[22] J.-H. He, "Preliminary report on the energy balance for nonlinear oscillations," Mechanics Research Communications, vol. 29, no. 2-3, pp. 107-111, 2002.

[23] A. G. Davodi, D. D. Ganji, R. Azami, and H. Babazadeh, "Application of improved amplitude-frequency formulation to nonlinear differential equation of motion equations," Modern Physics Letters B, vol. 23, no. 28, pp. 3427-3436, 2009.

[24] S. S. Ganji, D. D. Ganji, and S. Karimpour, "He's energy balance and He's variational methods for nonlinear oscillations in engineering," International Journal of Modern Physics B, vol. 23, no. 3, pp. 461-471, 2009.
[25] I. Mehdipour, D. D. Ganji, and M. Mozaffari, "Application of the energy balance method to nonlinear vibrating equations," Current Applied Physics, vol. 10, no. 1, pp. 104-112, 2010.

[26] H.-L. Zhang, "Periodic solutions for some strongly nonlinear oscillations by He's energy balance method," Computers and Mathematics with Applications, vol. 58, no. 11-12, pp. 2480-2485, 2009.

[27] D. Younesian, H. Askari, Z. Saadatnia, and M. KalamiYazdi, "Frequency analysis of strongly nonlinear generalized Duffing oscillators using HE's frequency-amplitude formulation and HE's energy balance method," Computers \& Mathematics with Applications, vol. 59, no. 9, pp. 3222-3228, 2010.

[28] H. Askari, Z. Saadatnia, D. Younesian, A. Yildirim, and M. Kalami-Yazdi, "Approximate periodic solutions for the Helmholtz-Duffing equation," Computers and Mathematics with Applications, vol. 62, no. 10, pp. 3894-3901, 2011.

[29] W. Q. Zhu, Random Vibration, Beijing Science Press, Beijing, China, 1992.

[30] K. Ito, "On stochastic differential equations," Memoirs of the American Mathematical Society, vol. 1951, no. 4, pp. 1-57, 1951. 


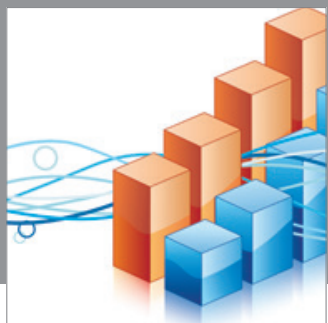

Advances in

Operations Research

mansans

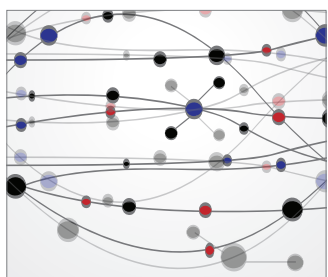

The Scientific World Journal
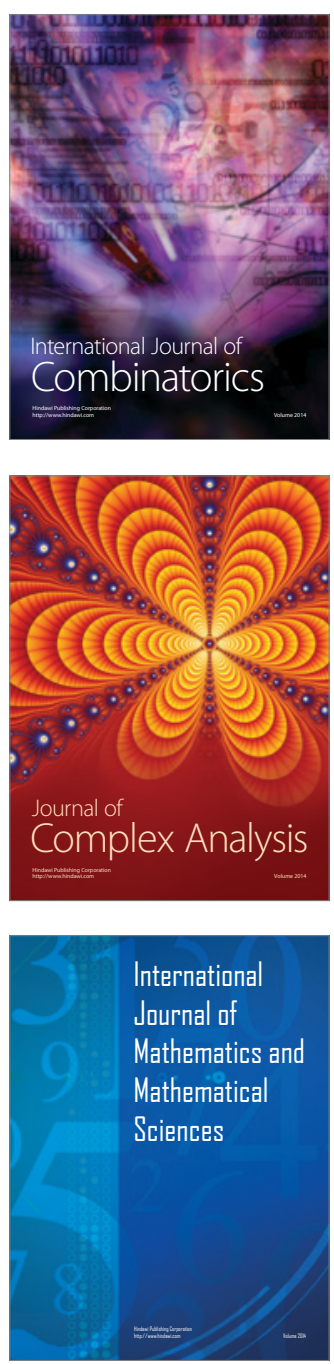
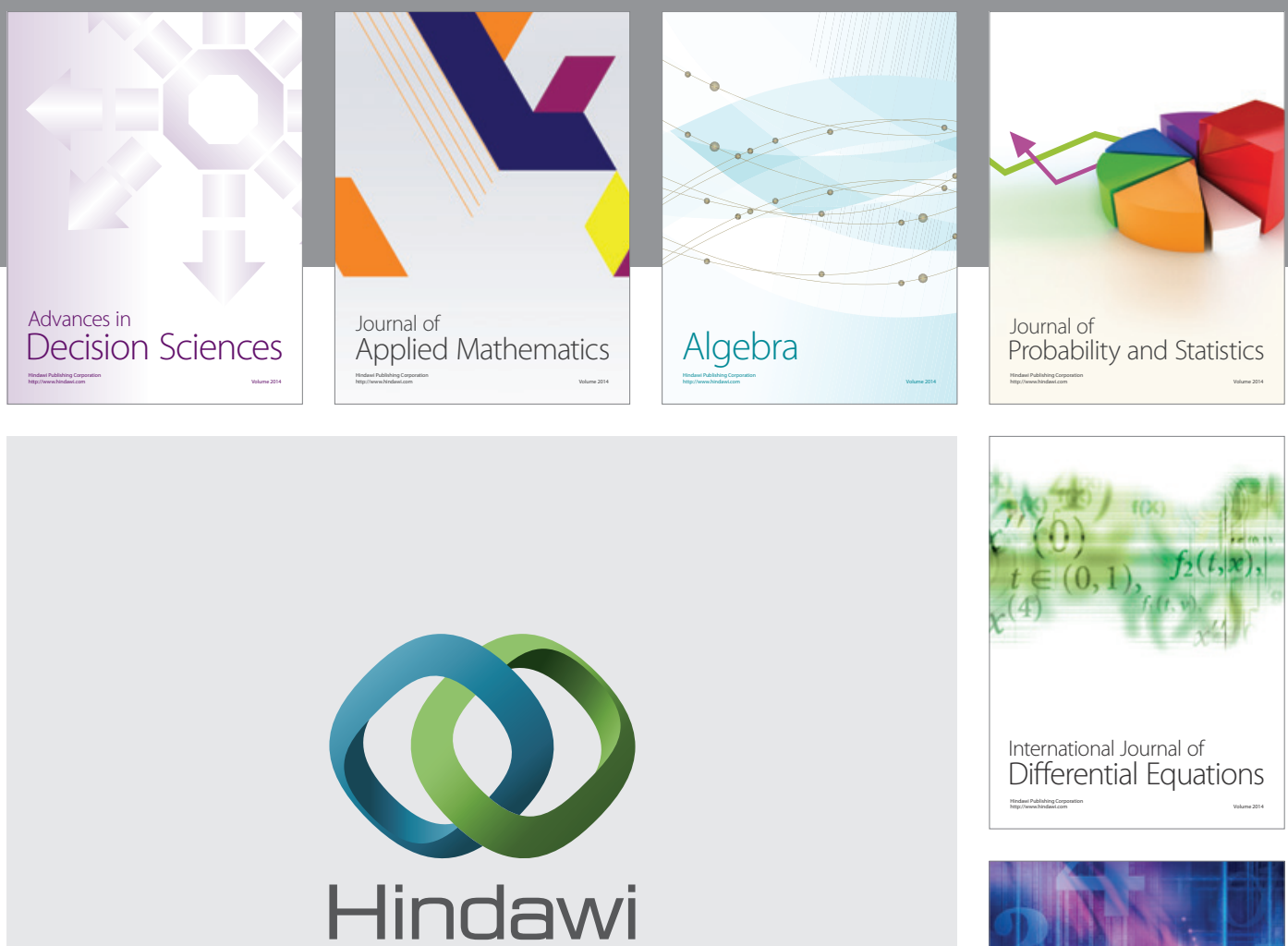

Submit your manuscripts at http://www.hindawi.com
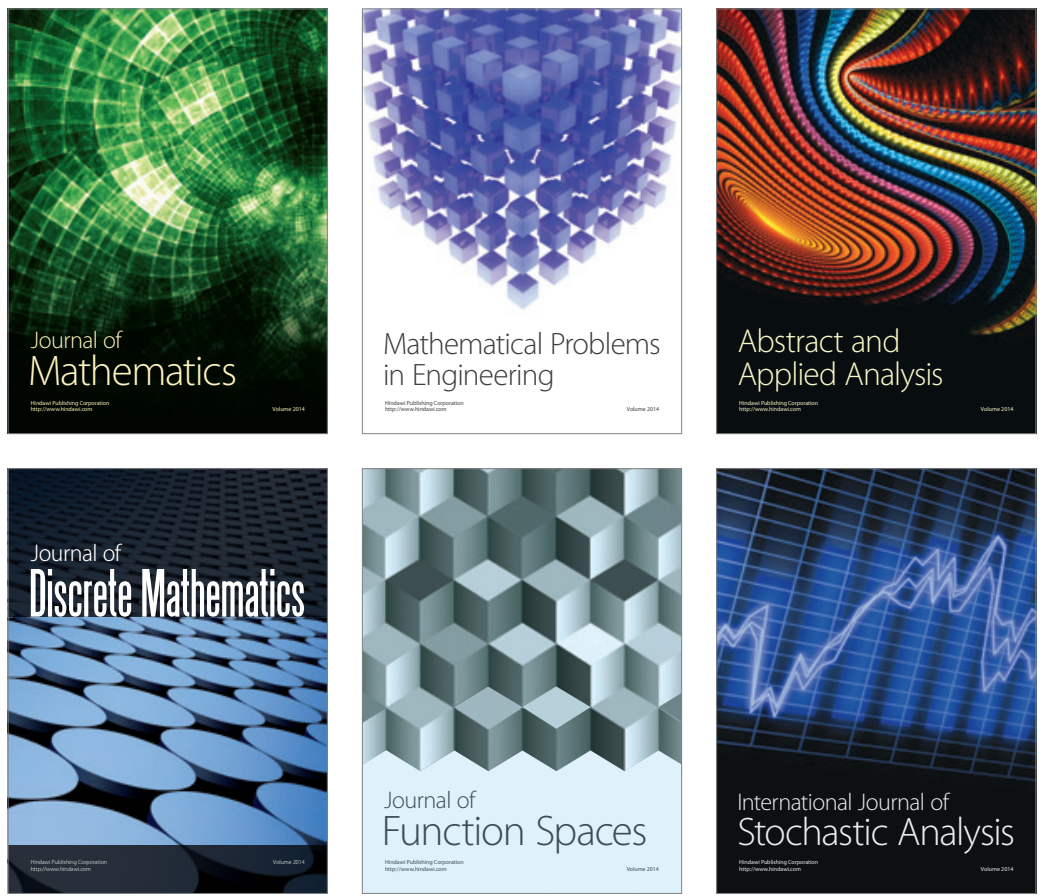

Journal of

Function Spaces

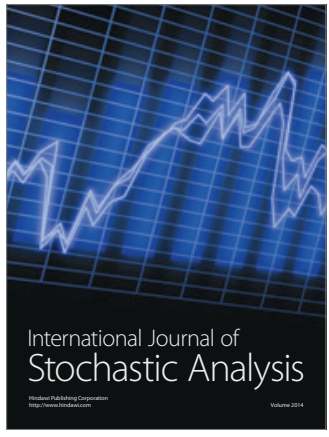

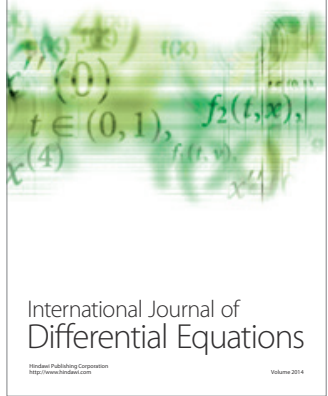
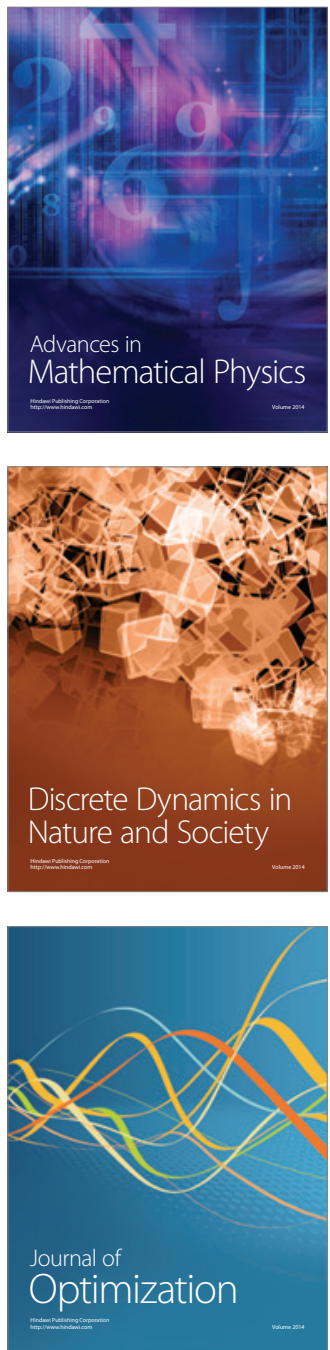